\title{
High-power, high-repetition-rate femtosecond pulses tunable in the visible
}

\author{
R. J. Ellingson \\ School of Applied and Engineering Physics, Cornell University, Ithaca, New York 14853 \\ C. L. Tang \\ School of Electrical Engineering, Cornell University, Ithaca, New York 14853
}

Received October 16, 1992

\begin{abstract}
We demonstrate a Ti:sapphire-pumped intracavity-doubled optical parametric oscillator (OPO) that generates a total of up to $240 \mathrm{~mW}$ of sub-100-fs pulses tunable in the visible. The OPO consists of a 1.5-mm-thick KTiPO (KTP) crystal configured in a ring cavity that is synchronously pumped by a self-mode-locked Ti:sapphire laser operating at an $81-\mathrm{MHz}$ repetition rate and 2.1-W average power, producing $115-\mathrm{fs}$ pulses at $\lambda=790 \mathrm{~nm}$.' Intracavity doubling of the OPO is accomplished by inserting a $47-\mu \mathrm{m}$-thick $\beta-\mathrm{BaB}_{2} \mathrm{O}_{4}$ crystal into an additional focus in the OPO cavity. We demonstrate continuous tuning of the second-harmonic output from 580 to $657 \mathrm{~nm}$. The potential tuning range of this intracavity-doubled KTP OPO is approximately 500 to $800 \mathrm{~nm}$.
\end{abstract}

Since the first demonstration of a high-repetition-rate femtosecond optical parametric oscillator (OPO) that was pumped intracavity of a colliding-pulse modelocked dye laser, ${ }^{1}$ there has been much interest in the development of the femtosecond OPO. The selfmode-locked Ti:sapphire (Ti:S) laser has led to a new level of performance in the high-repetition-rate femtosecond OPO. ${ }^{2-5}$ High-repetition-rate femtosecond OPO's have been demonstrated to cover the regions 0.755 to $1.04 \mu \mathrm{m}, 1.20$ to $1.40 \mu \mathrm{m}$, and 1.45 to 3.20 $\mu \mathrm{m} .{ }^{1-6}$ The Ti:S-pumped OPO offers several desirable operating characteristics: high output power, extensive tunability, nearly transform-limited pulses as short as $57 \mathrm{fs},{ }^{2}$ excellent stability, and low amplitude noise. In this Letter we demonstrate a Ti:Spumped ring-cavity OPO that is intracavity doubled to produce a stable $81-\mathrm{MHz}$ pulse train of $\sim 100-\mathrm{fs}$ pulses tunable from 580 to $657 \mathrm{~nm}$ with an average total power generated as high as $240 \mathrm{~mW}$ and pulse widths as short as 95 fs.

The Ti:S laser is configured in a standard linear cavity using an 18-mm-long titanium-doped $(0.1 \%)$ sapphire crystal. The pump beam $\left(11.5 \mathrm{~W} \mathrm{Ar}{ }^{+}\right.$, all lines) is focused using an $f=10 \mathrm{~cm}$ lens through one of the $r=10 \mathrm{~cm}$ Ti:S cavity mirrors. Dispersion compensation is achieved using SF-10 prisms spaced $45 \mathrm{~cm}$ apart; the laser is operated at an $81-\mathrm{MHz}$ repetition rate and produces 115 -fs pulses of $\sim 2.1-\mathrm{W}$ average output power at a center wavelength of $790 \mathrm{~nm}$.

The intracavity-doubled ring-cavity OPO is shown schematically in Fig. 1. An $r=25 \mathrm{~cm}$ dielectric mirror is used to focus the Ti:S laser beam, which is polarized along the KTP's $y$ axis, onto the $1.5-\mathrm{mm}$ thick KTP crystal. The KTP crystal, which is antireflection coated for $\lambda=1.3 \mu \mathrm{m}$, is cut at $\theta=45^{\circ}$ and $\phi=0^{\circ}$ for Type II phase matching $(o \rightarrow e+o)$ in the $x-z$ plane. The signal wave is resonated in a ring cavity using $r=15 \mathrm{~cm}$ mirrors for the KTP focus and $r=10 \mathrm{~cm}$ mirrors for the doubling crystal focus. The OPO mirrors are coated for a center wavelength of $1.3 \mu \mathrm{m}$. The OPO output coupler (OC) is either $1 \%$ at $1.3 \mu \mathrm{m}$ or a high reflecting mirror identical to the other cavity mirrors. The SF-14 prisms are spaced $20 \mathrm{~cm}$ tip to tip for dispersion compensation. The $47-\mu \mathrm{m}$-thick $\beta-\mathrm{BaB}_{2} \mathrm{O}_{4}$ (BBO) crystal, cut and polished in our laboratory, is Brewster-cut for Type I $(o+o \rightarrow e)$ phase matching at a wavelength of $850 \mathrm{~nm}$; orientation for phase matching the second-harmonic generation (SHG) at $1.3 \mu \mathrm{m}$ does not significantly increase the reflective loss suffered by the OPO fundamental. Unlike intracavity frequency doubling of a linear cavity, which results in dual output beams, the unidirectional ring

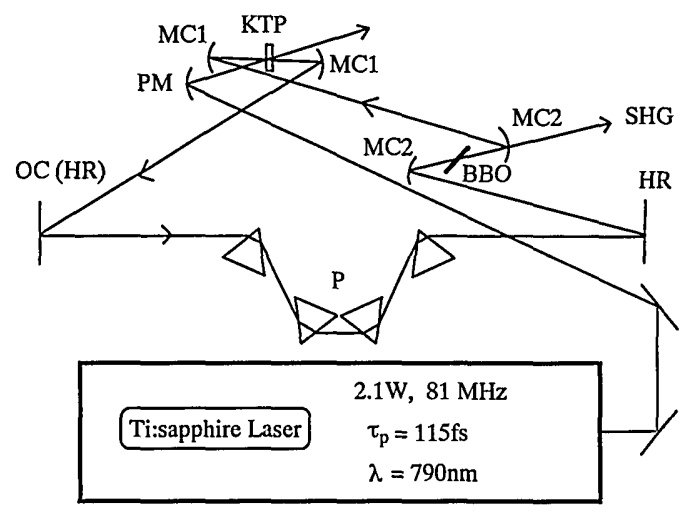

Fig. 1. Schematic of the Ti:sapphire-pumped intracavity-doubled KTP OPO. The Ti:sapphire pump is focused by the $r=25 \mathrm{~cm}$ pump mirror (PM) onto the 1.5-mm KTP crystal, which is cut for Type II phase matching in the positive region of the $x-z$ plane. The curved OPO high reflectors (MC1, MC2) surrounding the gain (KTP) and frequency-doubling (BBO) crystals have radii $r=15 \mathrm{~cm}$ and $r=10 \mathrm{~cm}$, respectively. The four-prism sequence (P) consists of two pair of $60^{\circ} \mathrm{SF}-14$ prisms spaced $20 \mathrm{~cm}$ tip to tip. The single SHG output is transmitted through the OPO high reflector (MC2) at the doubling focus. 
(a)

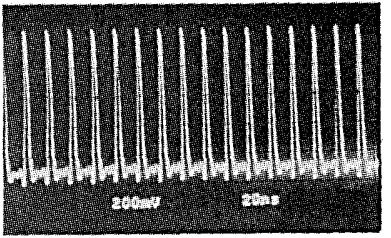

(b)

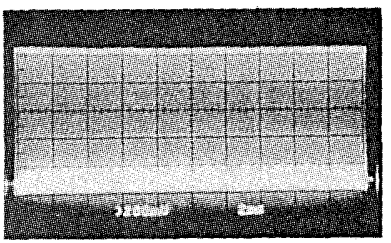

(c)

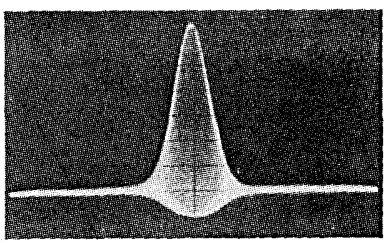

Fig. 2. Fast-photodiode oscilloscope trace showing the $\mathrm{SH}$ output of the intracavity-doubled OPO on time scales of (a) $20 \mathrm{~ns} /$ division and (b) $2 \mathrm{~ms} /$ division. The real-time autocorrelation in (c) shows a pulse width of $115 \mathrm{fs}$ (FWHM, sech ${ }^{2}$ fit) at $\lambda=647 \mathrm{~nm}$, corresponding to $240 \mathrm{~mW}$ of total power generated in the second harmonic.

cavity gives a single output for the second-harmonic (SH) beam.

Alignment of the intracavity-doubled OPO is similar to that of Ref. 5. Initial cavity alignment is performed with the additional frequency-doubling focus inserted but without either the $\mathrm{BBO}$ or prism sequence inserted; after the OPO is optimized, the prism sequence is inserted, the cavity length adjusted, and multipass signal maximized to regain oscillation. The OPO is again optimized, and the OPO signal beam output is used to orient the BBO crystal for phase matching at Brewster's angle. Subsequent insertion of the BBO into the additional focus does not destroy the multipass signal through the $1 \%$ output coupler, and regaining oscillation is straightforward.

When pumping with $2.1 \mathrm{~W}$, the intracavity-doubled OPO with the $1 \%$ OC produces an output of approximately $80 \mathrm{~mW}$ in the OPO signal beam at 1.3 $\mu \mathrm{m}$ and $115 \mathrm{~mW}$ of total $\mathrm{SH}$ light. As discussed further below, the main output of the $\mathrm{SH}$ through the OPO mirror is reduced by the Fresnel reflection off the $\mathrm{BBO}$, imperfect transmission through the OPO mirror, and loss from the collimating lens. We replace the $1 \% \mathrm{OC}$ with a high reflector (HR) to generate the highest SH power. In this configuration, after adjusting the intracavity prisms to yield the shortest SH pulses, we generate up to $240 \mathrm{~mW}$ of SH light at $\leq 115$-fs pulse width; we measure a 95-fs pulse width using a two-prism sequence (SF-14 glass, spaced $17 \mathrm{~cm}$ apart) to remove any chirp on the pulses. Although the OPO cavity is not actively length stabilized (stabilization is straightforward ${ }^{\mathrm{I}}$ ), the SH pulse train exhibits excellent stability as shown in Figs. 2(a) and 2(b). Furthermore, as shown in the real-time interferometric autocorrelation of Fig. 2(c), the pulses are clean and quiet.

Pumping the OPO with 115-fs pulses from the Ti:S laser, typical OPO pulse widths measured from the beam transmitted through the $\mathrm{OC}$ range from $\sim 120$ to $\sim 170 \mathrm{fs}$, depending on the intraprism path length. The pulse width of the second-harmonic output measured directly is $115 \mathrm{fs}$; a pulse width of $95 \mathrm{fs}$ is measured after the two-prism sequence, which reduces the time-bandwidth product from 0.45 to 0.37 . The time-bandwidth product of the OPO signal fundamental pulses taken through the $\mathrm{OC}$ is 0.45 (sech ${ }^{2}$ fit). This suggests that the OPO fundamental pulse may also be slightly chirped. Study of the specific pulse-shaping effects of both the intracavity prisms and the intracavity crystals should be possible by comparing the autocorrelation data for OPO output taken before and after the intracavity prism sequence.

As mentioned previously, the OPO may be tuned by varying the pump wavelength. ${ }^{2,5}$ Here we angle tune the OPO by rotating the phase-matching angle of the KTP crystal. Tuning requires slightly adjusting one flat mirror, the KTP crystal, and the cavity length; the OPO continues to oscillate during these adjustments, which take just a few minutes. Tuning of the intracavity-doubled OPO is simplified when using an ultrathin BBO crystal. The Type I SHG tuning curve for $\mathrm{BBO}$ is multivalued; i.e., a given phase-matching angle generally matches two distinct wavelengths for SHG. The degenerate point for SHG in BBO occurs at $\lambda=1.47 \mu \mathrm{m}$, and the SHG group-velocity mismatch also goes to zero at $\lambda=1.47 \mu \mathrm{m}$. Interestingly, this simultaneous matching of the phase velocity and the group velocity at the degenerate point also occurs in $\mathrm{LiNbO}_{3}$, another crystal of the $3 \mathrm{~m}$ point group. Around this degenerate point in the range of $\sim 1.1$ to $1.8 \mu \mathrm{m}$, the SHG bandwidth for the $47-\mu \mathrm{m}$-thick BBO crystal becomes very large (as large as $\sim 1700$ $\mathrm{nm}$, extending asymmetrically from roughly 1.0 to

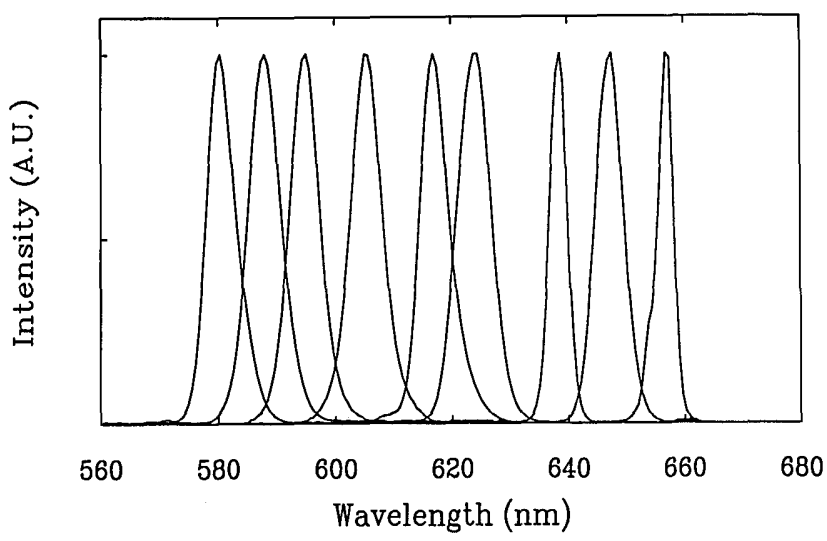

Fig. 3. Spectra showing the demonstrated tuning range of the intracavity frequency-doubled OPO from 580 to $657 \mathrm{~nm}$. Variation in the bandwidth may be explained by a slight length detuning of the cavity from optimal length matching. This effect, as well as adjustment of intraprism path length, has been observed to affect the autocorrelation pulse width. 
$2.7 \mu \mathrm{m})$ as the $\operatorname{sinc}^{2}\left(\Delta k l_{c} / 2\right)$ profiles for the two phase-matched wavelengths merge and cross. Our observations agree: e.g., after tuning the OPO from 1.3 to $1.2 \mu \mathrm{m}$, rotating the phase-matching angle has no noticeable effect on the conversion efficiency. Thus, when using a thin BBO crystal, tuning of the OPO does not require adjusting the phase-matching angle of the BBO. Representative spectra of the $\mathrm{SH}$ output are shown in Fig. 3.

Operating the intracavity-doubled OPO with the $1 \% 1.3-\mu \mathrm{m} \mathrm{OC}$, we measure the intracavity circulating power of the OPO fundamental to be $8 \mathrm{~W}$, which yields a SHG conversion efficiency of $\sim 1.4 \%$. Based on this measurement, we estimate that replacing the $1 \%$ OC with the high reflector increases both the intracavity circulating power and the SHG conversion efficiency to $\sim 11.5 \mathrm{~W}$ and $\sim 2.1 \%$, respectively. Use of a slightly thicker doubling crystal should facilitate the production of good power in the $\mathrm{SH}$ when lower Ti:S pump powers are used. The phase-matching bandwidth of even a $300-\mu \mathrm{m}$ BBO crystal would permit rotation-free phase matching from $\sim 1.2$ to 1.6 $\mu \mathrm{m}$, covering the $600-$ to $800-\mathrm{nm}$ range in the visible and near infrared.

Although the Brewster-cut BBO crystal minimizes Fresnel loss to the OPO fundamental, this configuration results in the $s$-plane-polarized SH beam's suffering a $21 \%$ Fresnel loss at the BBO exit surface. Use of a normal-incidence-cut 1.3- $\mu \mathrm{m}$ antireflectioncoated BBO crystal in place of the Brewster-cut piece would reduce the loss of the SH to $\leq 6 \%$. The only output coupling inefficiency for the $\mathrm{SH}$ beam is the transmission through the OPO high reflector. Our OPO mirrors transmit $\sim 80 \%$ of the $\mathrm{SH}$, and specially designed single-stack mirrors can increase this transmission to $\sim 90 \%$. Our current configuration, with losses of $21 \%$ at the BBO exit surface, $20 \%$ at the OPO HR, and $8 \%$ at the collimating lens, transmits $\sim 140 \mathrm{~mW}$ of the $240 \mathrm{~mW}$ total SH produced.

The nonlinear loss ( $2 \%$ total loss) due to the SHG in the intracavity-doubled OPO is not believed to play a major role in the shaping of the OPO fundamental pulse. The pulse broadening due to linear dispersion of such a thin BBO crystal is insubstantial. Thus it is expected that shorter Ti:S pump pulses obtained by reducing third-order dispersion ${ }^{7,8}$ will yield shorter OPO SHG pulses.

When weighting the benefits of doubling external or internal to the OPO cavity, one must consider both the advantages and disadvantages of each scheme. Extracavity doubling of the OPO has the advantage of simpler alignment, but presents the disadvantages of relatively low power (tens of milliwatts should be possible) and poor mode quality for the $\mathrm{SH}$ owing primarily to the angular acceptance limitations of using a thicker crystal. Conversely, intracavity doubling achieves approximately an order-of-magnitude greater power in the SH than is possible by extracavity doubling of current OPO output powers. The conversion efficiency is much greater intracavity since the power is $\sim 100$ times greater. Furthermore, the transverse mode of the OPO is an exceptionally pure, round $\mathrm{TEM}_{00}$ (achievable regardless of the transverse mode of the Ti:S pump laser), and aside from slight irregularity due to BBO surface imperfections, this $\mathrm{TEM}_{00}$ mode is imparted to the frequency-doubled beam.

We observe that intracavity-doubling with a thin BBO crystal does not add significantly to the OPO's complexity, nor does it reduce its stability. The linear loss of the additional focus and doubling crystal is estimated to be $\leq 0.5 \%$, and the OPO's tuning range is not affected by the presence of the BBO; in addition, use of a reasonably thin $\mathrm{BBO}$ crystal $(\leq 300 \mu \mathrm{m})$ permits hands-off phase matching over the tuning range of the OPO.

In conclusion, we have used an $\mathrm{Ar}^{+}$-pumped modelocked Ti:S laser pumping an intracavity-doubled OPO to generate tunable $\sim 100$-fs pulses over the range of 580 to $657 \mathrm{~nm}$, with a potential tuning range of $\sim 500$ to $800 \mathrm{~nm}$. We have demonstrated what we believe is an important source for highrepetition-rate femtosecond pulses, tunable in the visible, with high average power, short pulse width, excellent spatial mode quality, high peak power, and excellent stability. As extensions of this research, we plan to extend the demonstrated tuning range of the intracavity-doubled OPO to the shortest possible wavelengths by using a shorter-wavelength Ti:S pump beam and OPO mirrors that extend down to $\sim 1.05 \mu \mathrm{m}$. We also plan to replace the SF-10 prisms of the Ti:S laser with low-dispersion prisms and optimize the pulse-shaping parameters both of the pump source and within the OPO cavity to investigate short-pulse operation of the OPO.

We thank Randall Lane for cutting the BBO crystal and Mike Watts of Spectra-Physics Lasers, Inc., for his contribution to this Letter. This research was supported by the Joint Services Electronics Program and the National Science Foundation.

\section{References}

1. D. C. Edelstein, E. S. Wachman, and C. L. Tang, Appl. Phys. Lett. 54, 1728 (1989); E. S. Wachman, D. C. Edelstein, and C. L. Tang, Opt. Lett. 15, 136 (1990); E. S. Wachman, W. S. Pelouch, and C. L. Tang, J. Appl. Phys. 70, 1893 (1991).

2. W. S. Pelouch, P. E. Powers, and C. L. Tang, in Digest of Conference on Lasers and Electro-Optics (Optical Society of America, Washington, D.C., 1992), paper CPD14, p. 27.

3. G. Mak, Q. Fu, and H. M. van Driel, in Digest of Conference on Lasers and Electro-Optics (Optical Society of America, Washington, D.C., 1992), paper CWD1, p. 236.

4. Q. Fu, G. Mak, and H. M. van Driel, Opt. Lett. 17, 1006 (1992); G. Mak, Q. Fu, and H. M. van Driel, in Digest of Ultrafast Phenomena VIII (Ecole Nationale Superieure Technique et Avancée, Paris, 1992), p. 394.

5. W. S. Pelouch, P. E. Powers, and C. L. Tang, Opt. Lett. 17, 1070 (1992); presented at International Quantum Electronics Conference, Vienna, 1992.

6. G. Mak, Q. Fu, and H. M. van Driel, Appl. Phys. Lett. 60, 542 (1992).

7. C.-P. Huang, Ch. Spielmann, T. Brabec, E. Wintner, and A. J. Schmidt, Opt. Lett. 17, 204 (1992).

8. B. Proctor and F. Wise, Opt. Lett. 17, 1295 (1992). 